\title{
Influence of Testing Method on the Course of Anisotropy Coefficient
}

Josef Bradac, Jiri Sobotka

Department of Mechanical and Electrical Engineering, SKODA AUTO Vysoká škola o.p.s. Na Karmeli 1457, 29101 Mladá Boleslav. Czech Republic. E-mail: josef.bradac@savs.cz, jiri.sobotka@savs.cz

The anisotropy coefficient $r_{\alpha}$ [1] represents one of the main aspects of the materials deformation behavior, in addition to the basic material characteristics (such as e.g. yield strength, tensile ultimate strength, total ductility, uniform ductility). According to the standard, its value is calculated at a specific deformation value using the initial and actual measured length and width. However, modern materials testing methods offer the possibility to determinate its size throughout the deformation process that is however limited by the uniform ductility due to neck formation. In this paper, two such "modern" approaches are compared, where the values of anisotropy coefficient calculated according to the standard (i.e. just in one deformation value) are used as etalon. As these new testing methods, a mechanical strain gauge was used to determine the anisotropy coefficient throughout the whole static tensile test and optical contact-less deformation measurement system as well. Thus as the aim there was not only to measure and compare the anisotropy coefficient values courses during the static tensile test up to the uniform ductility, but also to compare them with the results measured by standard just in one deformation value. Possible utilization of these data can be found in the numerical simulation software.

Keywords: Anisotropy Coefficient, Deformation, Contact-less Optical Measurement, Aluminium Alloy, Tensile Test

\section{Introduction}

Beside strength and formability properties (e.g. yield strength, ultimate strength or total ductility), isotropy and anisotropy represents another very important material characteristics, because they describe general behaviour of material. Isotropic behaviour means that properties are direction independent. On the contrary, anisotropic behaviour means that properties are direction dependent. Quite a lot of different parameters can influence isotropic/anisotropic behaviour of materials. First of all, there are crystal lattices (for metals body centered cubic lattice, face centered cubic lattice and hexagonal closepacked lattice) that reveal at least different distances between atoms along different planes and directions. That is why monocrystalline structures always have a high level of anisotropy. In the case of polycrystalline materials is situation a little bit more complicated, because already own processing of metals (e.g. by forging or rolling), thermal treatment or just plastic deformation can greatly influence mechanical properties anisotropy. Thus they are preferred orientations or crystallographic textures in the material structure. E.g. slip on some slip planes can cause contraction in the plane of the sheet, but no thinning and that is why a useful parameter to quantify amount of plastic strain anisotropy was needed. Mathematically can be such behavior described by magnitude of so-called anisotropy coefficient (termed also as Lankford parameter) $r_{\alpha}[1]$, where $\alpha$ means angle with respect to the rolling direction. Generally, at least three angles are used: $0^{\circ}, 45^{\circ}$ and $90^{\circ}$ - always regarding $\mathrm{RD}[1,2,3]$.

The major aim of this paper was to determine values of the anisotropy coefficient $r_{\alpha}$ by the different approaches. At first, there were done "conventional" measurements. It means that specimens were elongated to require deformation and change of width and length was measured manually (conventional "point" method no. 1) or it was measured by the extensometer during the whole static tensile test (conventional "process" method no. 2). In addition to that, two optical methods were also used one via computing the whole front surface of tested specimen (photogrammetric "area" method no. 3) and another uses just change of distances between points (photogrammetric "points" method no. 4).

In the experimental part was used aluminium alloy AA6111 of thickness $0.9 \mathrm{~mm}$. Its producer is company AMAG Austria Metall AG. It contains a significant addition of copper (up to $0.9 \mathrm{wt} \%$ ) to make it stronger [1]. Before the own measurement of anisotropy coefficient, static tensile test was performed for all basic angles regarding RD. Thus there was carried out static tensile test at room temperature, strain rate $\mathrm{c}=0,002 \mathrm{~s}^{-1}$ and at directions $0^{\circ}, 45^{\circ}$ and $90^{\circ}$ regarding $\mathrm{RD}$. In Tab. 1 are summarized the basic mechanical properties of tested aluminium alloy AA6111.

Tab. 1 Static tensile test - basic material characteristics of AA6111

\begin{tabular}{|c|c|c|c|c|c|}
\hline \multicolumn{2}{|c|}{ Aluminium alloy AA6111 } & \multicolumn{2}{|c|}{ Strength properties } & \multicolumn{2}{|c|}{ Formability properties } \\
\hline \multicolumn{2}{|c|}{ Basic mechanical properties } & $\begin{array}{c}\text { Proof yield } \\
\text { strength }\end{array}$ & $\begin{array}{l}\text { Ultimate } \\
\text { strength }\end{array}$ & $\begin{array}{l}\text { Uniform } \\
\text { ductility }\end{array}$ & $\begin{array}{c}\text { Total } \\
\text { ductility }\end{array}$ \\
\hline Rolling direction $\left[{ }^{\circ}\right]$ & $\begin{array}{c}0^{\circ} \\
45^{\circ} \\
90^{\circ} \\
\end{array}$ & $\begin{array}{l}141.8 \\
134.4 \\
133.0\end{array}$ & $\begin{array}{l}259.1 \\
251.6 \\
248.8\end{array}$ & $\begin{array}{l}21.32 \\
24.19 \\
23.42\end{array}$ & $\begin{array}{l}26.81 \\
25.72 \\
27.62\end{array}$ \\
\hline
\end{tabular}




\section{Anisotropy coefficient - measurement by the "conventional" methods (no. 1 and no. 2)}

Anisotropy coefficient $r_{\alpha}$ [1] is basically measured at tensile test, because of the uni-axial stress state (see Fig. 1 ). Thus there is only major stress $\sigma_{1}$, which means that $\sigma_{1}$ equals effective stress $\sigma_{\text {eff. }}$ In light of the strain state (also shown in Fig. 1) is valid that:

Where:

$$
\varphi_{2}=\varphi_{3}=-\frac{\varphi_{1}}{2}
$$
[1],

$\varphi_{1}$ - true strain in the length direction (major strain) [1],

$\varphi_{2}$ - true strain in the width direction (minor strain)

$\varphi_{3}$ - true strain in the thickness direction[1].
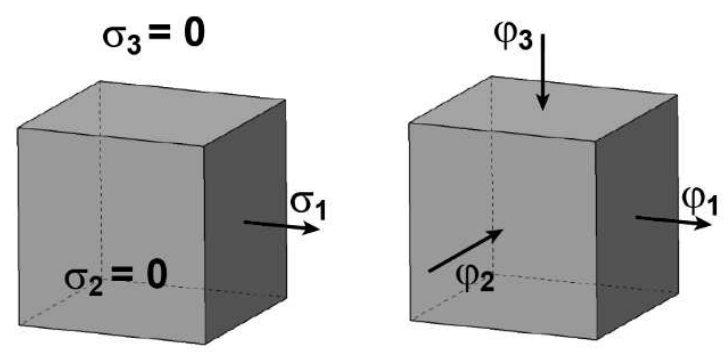

Fig. 1 Static tensile test - stress state (left) and strain state (right)

Anisotropy coefficient $r_{\alpha}$ [1] generally represents a useful parameter to quantify amount of plastic strain anisotropy and that's why it is mathematically computed according to following equation:

$$
r_{\alpha}=\frac{\varphi_{2}}{\varphi_{3}}
$$

Because it is sometimes quite difficult to measure thickness (or more precisely change of thickness), a modification of equation 2, arising from the constant volume law, is often used as following:

$$
r_{\alpha}=\frac{\varphi_{2}}{\varphi_{3}}=\frac{\varphi_{2}}{-\left(\varphi_{1}+\varphi_{2}\right)}=\frac{\ln \left(\frac{w}{w_{0}}\right)}{-\ln \left(\frac{L \cdot w}{L_{0} \cdot w_{0}}\right)}=\frac{\ln \left(\frac{w_{0}}{w}\right)}{\ln \left(\frac{L \cdot w}{L_{0} \cdot w_{0}}\right)}
$$

Where:

$\mathrm{L}_{0}, \mathrm{~L}-$ initial and final (or actual) gage length[mm], $\mathrm{w}_{0}, \mathrm{w}$ - initial and final (or actual) gage width $[\mathrm{mm}]$.

Two measurement methods were used in the "conventional" approach - method no. 1 (termed as "point" method, because anisotropy coefficient was computed only in one point) and method no. 2 (termed as "process" method, because testing jig makes possible to measure anisotropy coefficient during the whole process of deformation).

Conventional "point" method no. 1 is the classic one. In this case is specimen deformed on the given value of strain. Such magnitude is always specified for a tested material and arises mainly from its total ductility. In this case of aluminium alloy AA6111 having total ductility about $25 \%$ were specimens firstly deformed on the magnitude of engineering strain equal $\varepsilon_{\text {eng }}=0.2$. After that there was measured final length (L) and final widths (w) in three different areas (average was computed after that) and together with initial length $\left(\mathrm{L}_{0}\right)$ and width $\left(\mathrm{w}_{0}\right)$, equation 3 was used to finally compute anisotropy coefficient $r_{\alpha}$ in this very one point.

Conventional "process" method no. 2 represents a tendency to mechanically measure anisotropy coefficient during static tensile test. Beside common gauge length is in this case tester equipped with the mechanical gauge width, which is able to measure actual width in every moment of static tensile test. Again, equation 3 was used, but now for the whole deformation process (static tensile test). In Tab 2 are compared results from these two "conventional" approaches. Even the second method ("process" one) is taken also right in one value $\left(\varepsilon_{\text {eng }}=0\right)$, just be able to compare them. Differences are quite small (cca 1\%), but note that "process" values are always higher

\begin{tabular}{|c|c|c|c|c|}
\hline \multicolumn{2}{|c|}{ Aluminium alloy AA6111 } & \multicolumn{3}{|c|}{ “Conventional" measurement - Eeng $=0.2$} \\
\hline \multicolumn{2}{|c|}{ Anisotropy coefficient $r_{\alpha}[1]$} & $\begin{array}{l}\text { Conventional "point" } \\
\text { method - no. } 1\end{array}$ & $\begin{array}{l}\text { Conventional "pro- } \\
\text { cess" method - no. } 2\end{array}$ & $\begin{array}{c}\text { Difference }[\%] \\
(\text { method no. } 1-100 \%)\end{array}$ \\
\hline \multirow{3}{*}{ Rolling direction $\alpha\left[^{\circ}\right]$} & $\mathbf{0}^{\circ}$ & 0.7802 & 0.7864 & $+0.79 \%$ \\
\hline & $45^{\circ}$ & 0.5272 & 0.5346 & $+1.40 \%$ \\
\hline & $90^{\circ}$ & 0.6593 & 0.6678 & $+1.23 \%$ \\
\hline
\end{tabular}
than "point" ones.

Tab. 2 "Conventional" measurement of the anisotropy coefficient $r_{\alpha}$

\section{Anisotropy coefficient - measurement by the contact-less optical method (no. 3 and no. 4)}

Another part of methods, which were used to measure the anisotropy coefficient $r_{x}$, arises from photogrammetry, i.e. measurement from photos - thus contact-less deformation measurement. In this case was used system MERCURY RT from the company Sobriety Ltd. The whole arrangement of optical device ( 2 cameras) that was used at static tensile test is shown in Fig. 2. Optical systems generally require quite time consuming preparation before the own measurement. In this case it was necessary to firstly degrease testing specimens, then apply on their surface stochastic pattern (via spraying the 
white background and small black dots) and finally to adjust own cameras by their focusing, setting the proper shutter time and applying relevant calibration device (e.g. by calibration panel or cross) to set so-called calibration volume within it control software ensures given accuracy, which mainly depends on the measuring distance.

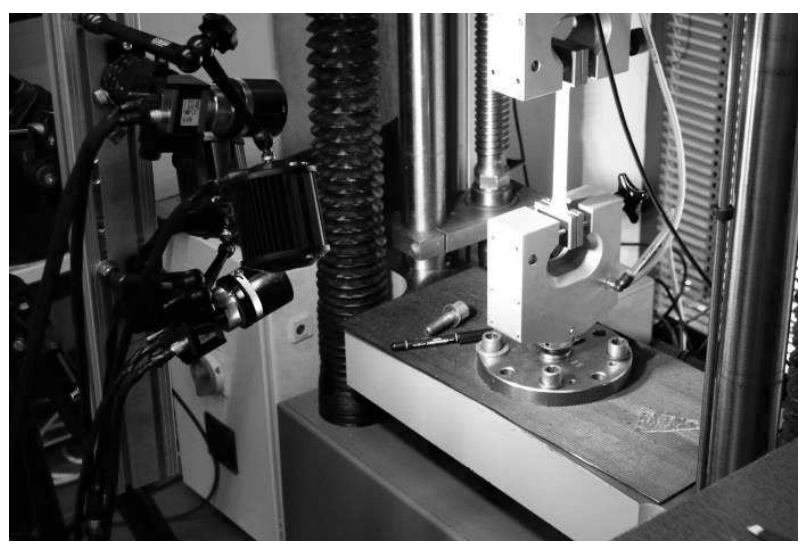

Fig. 2 Contact-less optical system at measuring the anisotropy coefficient $r_{x}$
After proper adjustment of cameras, there were performed just 3 tests for every rolling direction $\left(0^{\circ}\right.$, $45^{\circ}$ and $90^{\circ}$ ). In Fig. 3 is shown the environment of software MERCURY RT, which controls cameras and whole post-processing is done here. Two evaluation methods (named as "area" and "points") were used for this photogrammetric measurement. First method (no. 3) utilized computation of the almost whole front surface of the relevant testing specimen. Only two output channels were necessary to be used at this measurement - one for major strain $\varphi_{1}$ and second for minor strain $\varphi_{2}\left(\varphi_{3}\right.$ is taken from the constant volume low). Data from the whole surface can be subsequently for every stage taken as minimal, average or maximal values (average ones were used in this paper). Equation 2 was used to compute the anisotropy coefficient. Second method (no. 4) applied just points (thus "points" method) on the measured surface and as a result there were changes of distances between them. These points can be seen in Fig. 3, 4 and 5. There was always used just one longitudinal distance (A1) to measurement change of length and three transverse directions (A2, A3 and A4) to measure change of width in three zones. Equation 3 was finally used to compute the anisotropy coefficient.

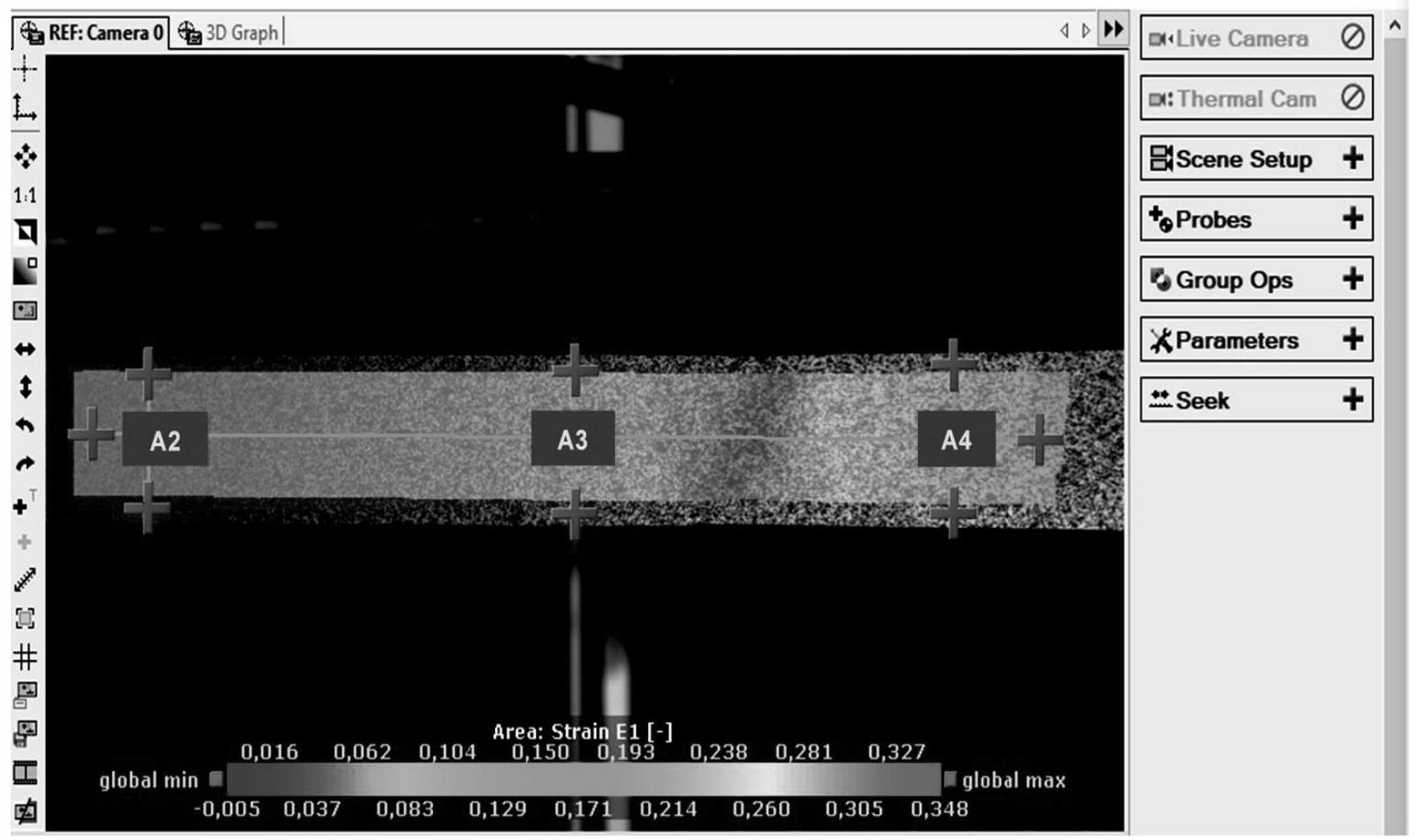

Fig. 3 Environment of the software MERCURY RT (in this case RD $90^{\circ}$ and stage 715 )

Both photogrammetric approaches ("area" - no. 3 and "points" - no. 4) are shown on this page. Fig. 4 shows stage 0 (without deformation) with applied both area for computation true strains (in this case for major strain $\varphi_{1}$ ) and points (shown in violet colour) with relevant lines: A1 for longitudinal direction and A2, A3, A4 for transverse directions.
In Fig. 5 is shown the same specimen as before (to be specific - rolling direction was $90^{\circ}$ ), only now it illustrates distribution of major strain $\varphi_{1}$ right before the fracture. Note the position of violet points and thus also lengths of the relevant lines (A1, A2, A3, A4), which also graphically illustrates the magnitude of deformation. 


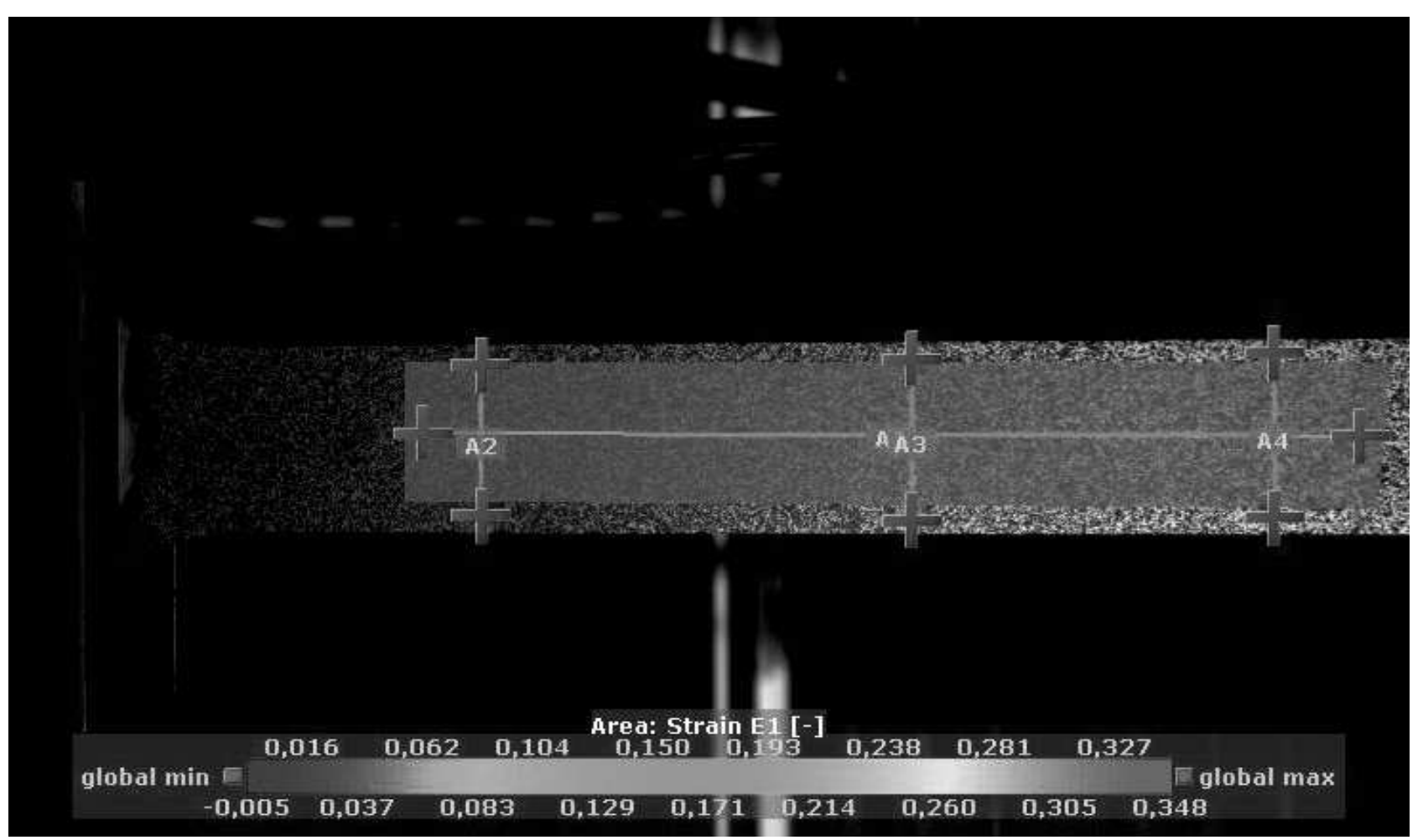

Fig. 4 Distribution of major strain $\varphi_{1}$ and position of measured points in stage 0

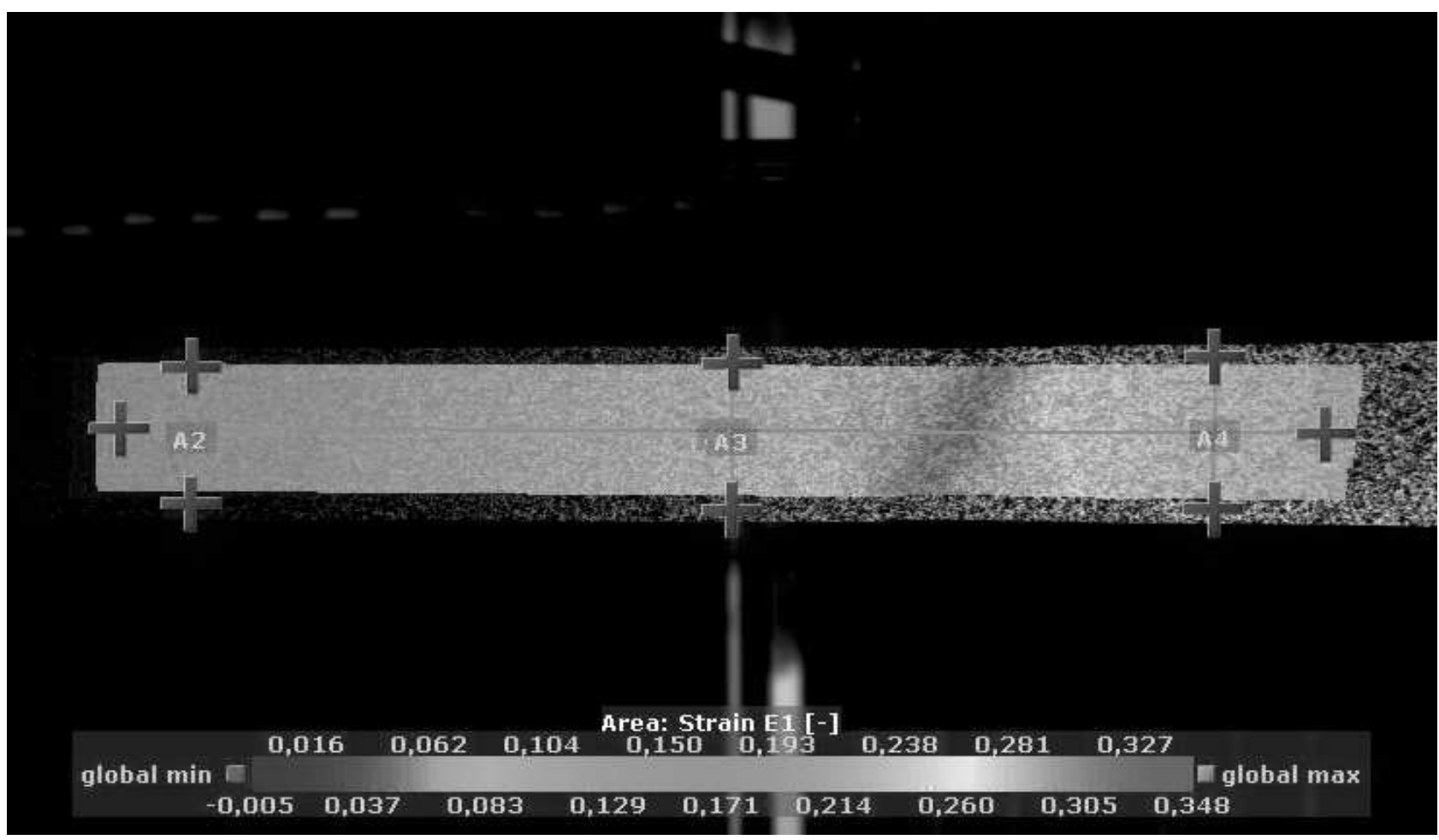

Fig. 5 Distribution of major strain $\varphi_{1}$ and position of measured points right before the fracture (stage 715)

From measured data were subsequently computed all required courses as anisotropy coefficient $r_{x}[1]$ vs. engineering strain $\varepsilon_{\text {eng }}[1]$. It was done almost directly in the case of "area" method (no. 3). There was needed only conversion from true strain to engineering strain. A little bit more complicated procedure had to be used in the case of "points" method (no. 4), because transverse values (width direction) were firstly statistically evaluated to apply average value in every moment of static tensile test. Fig. 6 shows such graphical comparison of these two photogrammetric methods for $\mathrm{RD} 90^{\circ}$. It is obvious that "points" method is quite very sensitive both at the beginning (probably due to low number of points - so very sensitive to initial adjustment) and at the end (influence of necking). 


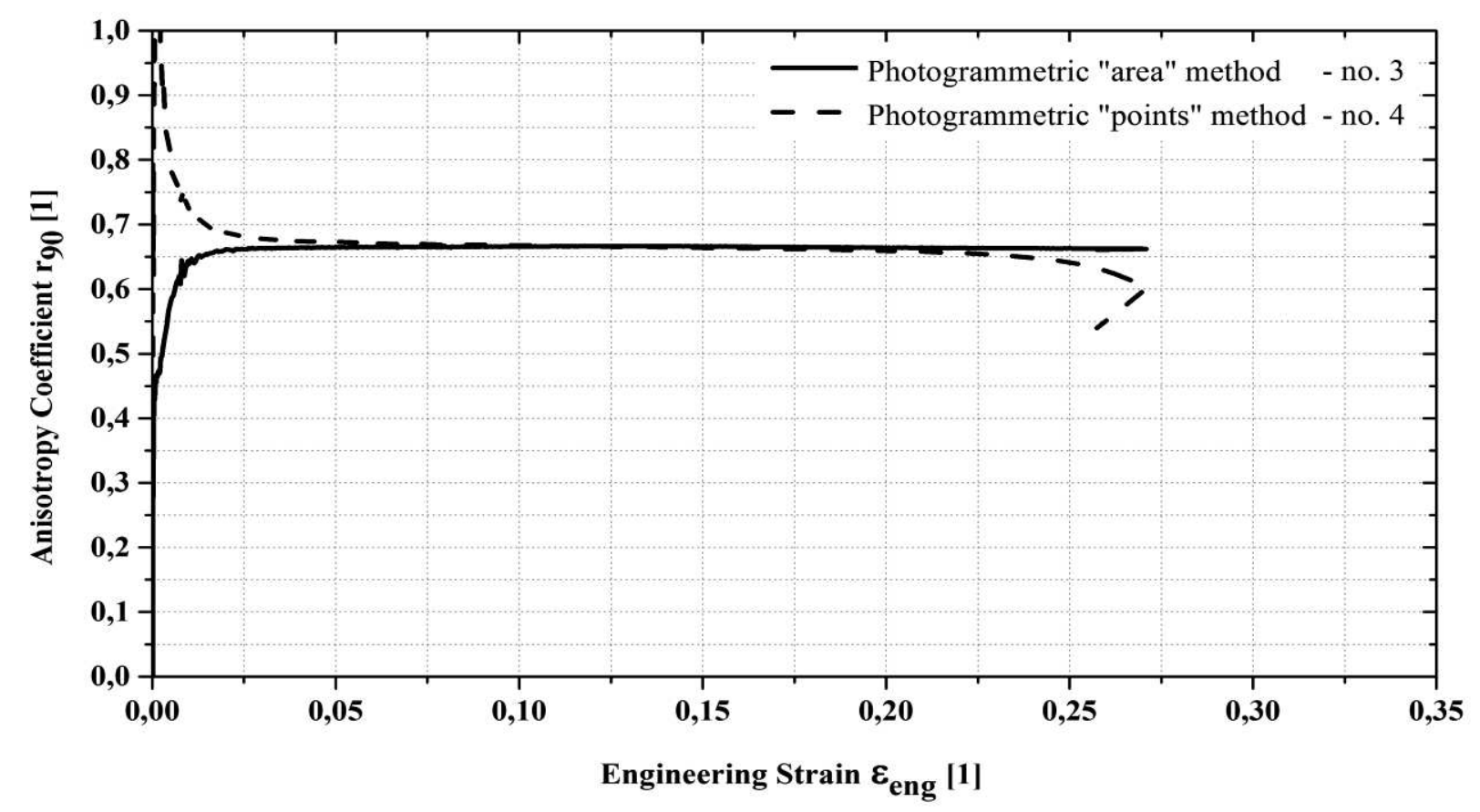

Fig. 6 Distribution of major strain $\varphi_{1}$ right before fracture

Because "points" method (no. 4) revealed the same shape of curves for all RD, it is not shown in the graphs below. Fig. 7 shows quite important comparison between photogrammetry "area" method (no. 3 - curves) and conventional "point" method (no. 1 - crosses). Vertical red dot line is for $\varepsilon_{\mathrm{eng}}=0.2$. There is evident good matching between them.



Fig. 7 Comparison between method no. 1 (crosses) and method no. 3 (curves)

\section{Conclusion}

There were several goals of this paper. As a major one, there was effort to carry out some different approaches how to measure the anisotropy coefficient $r_{\alpha}$ [1]. In addition to that, there was ambition to perform anisotropy measurement just by the contact-less optical system and via its comparison with the "conventional" approaches, to verify its usability for this kind of measurement. Because such approach can be sometimes applied right together with contact-less optical measurement of deformation on the surface of tensile test specimen, it can be used also as e.g. by-product from this type of measurement. As a tested material there was used aluminium alloy AA6111 of thickness $0.9 \mathrm{~mm}$. This representative of the $6 \mathrm{xxx}$ aluminium alloys series was chosen with respect to its wide utilization in the 
automotive industry.

In the experimental part were totally used 4 different

"Conventional" methods

Method no. 1

Method no. 2

"Photogrammetric" methods

Method no. 3

Method no. 4 types of measurements, which can be classified as following:

- "point" method

- specimens were deformed to one value of engineering deformation ( $(\varepsilon e n g=0.2$ ) and equation 3 was used

- "process" method

- anisotropy coefficient was computed in the course of whole static tensile test acc. to equation 3

- "area" method

- values of $\varphi 2$ and $\varphi 3$ were computed from the whole front surface of specimen and equation 2 was used

-"points" method

- from the computed front surface of specimen were taken just lines (A1 -A4) and equation 3 was used

is valid just for $\varepsilon_{\text {eng }}=0.2$. Comparison of the whole courses of anisotropy coefficient in dependence on deformation (here measured by method 2, 3 and 4) is going to be interest of the future research. On the other hand, already these results can be utilized in the numerical simulations as quite important input material data.

Fig. 8, where are compared values of anisotropy coefficient $r_{\alpha}$ for all RD and measured by these 4 methods. First two columns ("conventional" methods) have been already compared in Tab. 2. Variation of results is quite very low $\left(1.23 \%\right.$ for $0^{\circ}, 1.97 \%$ for $45^{\circ}$ and $1.32 \%$ for $90^{\circ}$ ), so these methods are comparable. But this

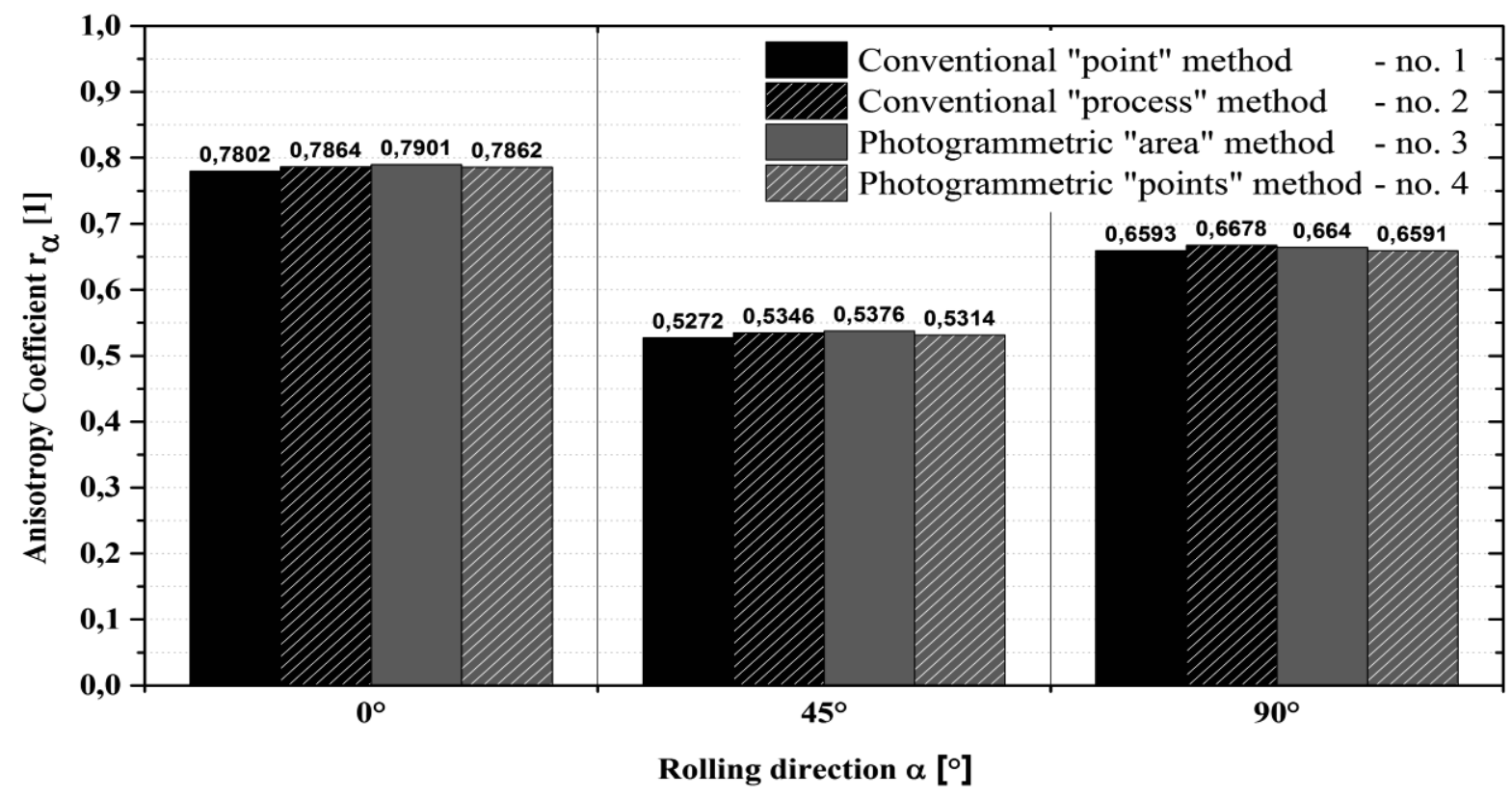

Fig. 8 Final comparison of all used measurement methods (no. 1 - 4) for engineering deformation $\varepsilon_{\text {eng }}=0.2$

\section{References}

[1] KUHN, H., D. MEDLIN, ed. (2000). ASM HANDBOOK Volume 8 - Mechanical Testing and Evaluation. ASM International, Materials Park.
[2] POLMEAR, I.(2006) Ligth Alloy - From traditional Alloys to Nanocrystals. Butterworth-Heinemann, Oxford.

[3] NESLUSAN, M. et al. (2016). Magnetic Anisotropy of Hard Milled Surfaces. Manufacturing Technology, Volume 16. pp. 1073-1078. 